\title{
INDICADORES DE RSC EN LA EMPRESA PERIODÍSTICA
}

\author{
Juan Luis Manfredi Sánchez \\ (IE Universidad) \\ juan.manfredi@ie.edu
}

\begin{abstract}
Resumen: El auge de la responsabilidad social corporativa (RSC, en adelante) se ha apoyado en los medios, que han incluido este elemento de la gestión empresarial en las secciones de economía y sociedad. Las tres principales empresas privadas españolas de televisión no han desarrollado una política de RSC adecuada a las necesidades y los criterios propios como sucede en otros sectores. EI análisis ofrece un bagaje pobre. Urge la definición de la especificidad de las empresas periodísticas, la elaboración de un catálogo de criterios para la medición e introducir elementos comparativos que sirvan como marco para las buenas prácticas.
\end{abstract}

Palabras clave: Empresa, televisión, responsabilidad social corporativa.

Abstract: The rise of corporate social responsibility (CSR) has been supported by the media, which have included this element of management in the news. The three Spanish top private television companies have not developed a CSR policy appropriate to the needs and standards of management and organization own business as in other sectors. The analysis of reports and annual reports provide a wealth poor. We need to define the specificity of the journalistic enterprise, developing a list of criteria and introduces comparative elements from public and private companies that serve as a framework for good practices.

Keywords: Media management, television, csr.

\section{LA RESPONSABILIDAD SOCIAL CORPORATIVA EN LA EMPRESA PERIO- DÍSTICA}

a responsabilidad social corporativa (RSC) es un concepto amplio, que abarca numerosas áreas de la gestión y la dirección de las empresas. La primera responsabilidad consiste en las obligaciones económicas como ser rentable, eficiente y generar beneficios. La segunda es la obediencia de la ley y del marco reglamentario vigente, mientras que la tercera se asienta sobre las responsabilidades éticas, esto es, la obligación de hacer lo que es correcto, justo y bueno, evitando el daño a terceros. La actitud empresarial trataría de minimizar las acciones que van en detrimento de los públicos y de la ciudadanía. Finalmente está la responsabilidad filantrópica que consiste en la buena ciudadanía, la contribución al crecimiento de la comunidad donde opera y la mejora de la calidad de vida. La suma de las cuatro responsabilidades conforma una visión global de la responsabilidad social corporativa (Carroll y Buchholz, 1999: 37). 
En cualquier caso, la propia definición de responsabilidad social o su ámbito de aplicación están directamente ligados a los contextos legales, culturales y económicos del sector al que nos refiramos. Las cuestiones sociales y medioambientales son mayoritarias, si bien cada sector económico o industrial debe atender a las especificidades derivadas de su actividad, su perfil, la historia o la situación de cada empresa.

Como consecuencia de este planteamiento, la empresa integra la RSC en la toma de decisiones, en las operaciones y en la creación de valor. La transparencia, el diálogo y el trabajo con el entorno que rodea la empresa son los valores de reconocido prestigio para la consecución de mejores productos o servicios, el incremento de la calidad y la excelencia, la mejora constante de los procesos y los resultados. Comprende un conjunto de acciones de carácter voluntario, que dirigen su atención no únicamente de los accionistas, sino hacia el conjunto de la sociedad, incluyendo los empleados, los consumidores, los gobiernos, los proveedores, los inversores, entre otros. Esta concepción precisa de la reflexión, la construcción y el desarrollo de las medidas que cada empresa considere adecuadas. La responsabilidad social no puede ser impuesta desde la Administración Pública, si bien ésta puede facilitar el proceso de adecuación y adaptación mediante incentivos fiscales o de otro corte, que generen certidumbre a la empresa. A riesgo de ser menos competitiva en el corto plazo, la firma que se comprometa con su entorno, gana en estabilidad e incrementa la confianza de los inversores. Esta estrategia opera en el largo plazo y no se reduce a la mejora de los resultados económicos, sino que se amplía a las áreas de utilidad y responsabilidad para con la sociedad.

El impacto de las decisiones empresariales en la sociedad tiene que evaluarse de acuerdo con sus consecuencias, sean intencionadas o contingentes. Siguiendo la literatura anglosajona, diferenciamos entre los shareholders (accionistas) y los stakeholders (interesados). Este enfoque reconoce las externalidades de cualquier empresa y la obligación que se deriva de ellos. Incluye a todos los actores propios y ajenos de la organización (clientes, usuarios, consumidores, proveedores, administraciones públicas, empleados, socios tecnológicos) y a la sociedad misma en tanto que receptora pasiva de las acciones de la empresa. Estos últimos tienen como meta el incremento de la utilidad social de la empresa. Esta hipótesis apela a la relación entre empresa y sociedad, de modo que establece una jerarquía de intereses, de obligaciones y de responsabilidades. Los diversos grados de responsabilidad y exigencia principian por aquellos económicos y legales, pero se amplían en la línea de los éticos y los filantrópicos de acuerdo con esta interpretación extensa de la responsabilidad. Cobra sentido la misión de la empresa, que se define como el objetivo último de la empresa. Los valores emanan de la misión, de modo que los beneficios económicos son resultado de la política de la compañía, pero no los principios rectores.

La gestión de responsabilidad se sustenta sobre la identificación de los grupos y los intereses afectados por la actividad de la empresa. En el caso de las em- 
presas periodísticas, los interesados son todos los ciudadanos que tienen acceso a la misma (cfr. los indicadores de consumo y ocio del Ministerio de Cultura, de la SGAE o de la Comisión Europea). Los intereses son complejos, pero pasan por el peso creciente de los medios en la gobernanza, en las elecciones y el proceso político general, en la educación y en las habilidades sociales, o en el consumo, entre otras. Las habilidades sociales son las capacidades de ejecución de las conductas aprendidas y que cubren las necesidades de comunicación social e interpersonal. Las habilidades, aprendidas en un contexto grupal, facilitan la respuesta a las situaciones y las exigencias sociales de forma efectiva.

La referencia internacional recurrente es el Pacto Mundial de las Naciones Unidas, que consiste en una iniciativa internacional propuesta por Naciones Unidas en el año 2000. El objetivo es la extensión del compromiso voluntario de las entidades y organizaciones en materia de responsabilidad social por medio de la implantación de diez principios relacionados con los derechos humanos, las relaciones laborales, la protección medioambiental y la lucha contra la corrupción. Involucra directamente a las organizaciones y las compañías que gestionan buena parte del impacto y el desafío de la globalización en materia social y medioambiental. Es de carácter abierto y ofrece un marco de trabajo y desarrollo de las iniciativas. En el marco empresarial, se encuentran las líneas directrices de la OCDE aprobadas en 2002. Son recomendaciones voluntarias que los gobiernos de los países miembros han lanzado a las empresas, con especial atención a las multinacionales. Afectan a la protección y defensa de los derechos humanos, al respeto a las relaciones laborales, la protección del medio ambiente y fomento de la gobernanza pública. Dichas directrices sirven a la empresa como referente de normas y principios de buena práctica corporativa que son compatibles y aplicables dentro de la legislación vigente.

\subsection{La especificidad de la empresa periodística}

La autorreferencialidad, el auge del infoentretenimiento y la doble condición de las empresas periodísticas generan un marco de trabajo muy interesante. Por un lado, la función informativa les confiere una posición de relevancia y estimula la idea de que son corresponsables en la creación de la agenda temática. La influencia no consiste en la elaboración de los juicios, sino en la exposición reiterada de unos temas frente a otros. La radio, la prensa, la televisión y, cada vez más, Internet determinan la agenda temática y son responsables de la introducción y la exclusión de las cuestiones de la opinión pública. En este sentido, crece la atención de los medios por la responsabilidad social, que aparece mayoritariamente vinculada a la sección de economía y a grandes empresas, no a pymes u organismos del tercer sector. La indefinición del concepto y el uso interesado del mismo provoca que bajo la etiqueta "RSC" se presenten noticias de buen gobierno y protección del medio ambiente, pero se excluyan las políticas laborales y de igualdad. El exceso de información positiva, la vinculación de la noticia a un pseudoevento y la empresa como fuente única de la misma acercan las noticias de RSC a la publicidad corporativa y reducen la credibilidad de las 
noticias. Cumplen la obligación de informar, lo que no significa que se produzcan cambios o mejoras en el gobierno corporativo, ya que no hay un análisis crítico y cualitativo de las informaciones, sino una presentación suave de las buenas maneras de la gestión y administración de una empresa.

Por otro, son empresas que responden ante la sociedad y que deben desarrollar un entorno sectorial adecuado al escenario actual que valora la cultura y las prácticas responsables. Está por determinar y evaluar la ventaja competitiva que se extrae del comportamiento socialmente responsable, si bien ya se apunta en los primeros indicadores de valoración bursátil y en la gestión de la relación con los inversores. La salida a Bolsa y el crecimiento de los inversores institucionales, así como fondos de todo tipo, han consolidado el peso de la industria mediática. Entre 1999 y 2004 la inversión pública en Bolsa se ha incrementado un 65,20\% en la industria de la prensa estadounidense (Soloski, 2005: 70). En nuestro caso, en 1995 las cadenas privadas Antena 3, Telecinco y Canal Plus ofrecieron los primeros resultados económicos positivos, sin necesidad de que todas fueran líderes de audiencia en términos absolutos (Cubeles, 2002: 31). Este proceso corre paralelo con la internacionalización de las estrategias, los mercados y las estructuras, dentro del proceso de desregulación (el estado facilita la entrada de más actores en el sector audiovisual y expande las dinámicas del mercado), de globalización (el mercado es único, es mundial y funciona en tiempo real), de internacionalización, de integración (la multiplicación de venta permite una línea continua de gestión desde la creación del producto a su puesta en valor en Internet) y de convergencia.

La inversión institucional tiene como finalidad primera el incremento del valor de la acción y pone en la encrucijada las decisiones periodísticas, informativas y comunicativas. ¿Podría una empresa de televisión mantener una red de corresponsales propia, si no genera beneficios a corto plazo? El estudio del profesor John Soloski sobre los grupos mediáticos de comunicación cotizados confirma que este tipo de inversión está interesada en el corto plazo, así como el ROE (ratio que mide la rentabilidad que obtienen los accionistas de los fondos invertidos en la compañía) y no se preocupa por el conjunto de prácticas periodísticas que desarrollen las redacciones o los contenidos emitidos. Recientemente, The New York Times ha decidido la retirada progresiva de su pool de corresponsales en Iraq ante el descenso del interés público en esa guerra y el previsible auge del enfrentamiento en Afganistán. En 2009 la decisión se traducirá en el ahorro de costes por valor de tres millones de dólares. El control financiero prima sobre las decisiones periodísticas, de modo que la redacción es la prima víctima de los recortes en tiempos de crisis. El modelo económico de empresa cotizada ha conducido a un descenso en los salarios, en la orientación hacia al anunciante, en la definición de las audiencias en términos comerciales y en la reducción del interés por los ingresos por circulación. Como titulaba el número de invierno de la revista FAPE, estamos en un proceso negativo de reducción de profesionales contratados, pasando "del becario precario a la redacción precaria". 
Esta crisis del sistema informativo industrial se ha cobrado numerosas víctimas. Entre 2000 y 2007 se ha suprimido uno de cuatro empleos en el sector de los medios tradicionales en Estados Unidos, al tiempo que ha crecido el sector de marketing y publicidad e Internet. La reducción del número de periodistas y el enfoque industrial, léase publicitario, ha perjudicado la percepción de calidad por parte de los lectores y espectadores. En la encuesta realizada por WeMedia/Zogby Interactive, el $67 \%$ de los ciudadanos estadounidenses creen que el periodismo que actualmente se realiza está alejado de sus intereses. Como consecuencia del cambio, la mayor parte de la población ha abandonado los medios tradicionales en beneficio de Internet como fuente primaria de información y conocimiento. El porcentaje crece hasta el 55\% entre los nativos digitales de entre 18 a 29 años. Resulta positivo que casi el $70 \%$ de la muestra considere que el periodismo es positivo para su calidad de vida individual y colectiva, aunque no estén satisfechos con la calidad.

\section{BENCHMARKING DE BUENAS PRÁCTICAS}

Las empresas periodísticas se sustentan sobre un modelo que puede conducir al conflicto de interés, que consiste en la capacidad que tiene un elemento ajeno a la empresa de intervenir en el desarrollo corporativo o en la toma de decisiones. La economía del medio se sustenta sobre las aportaciones de capital y los ingresos procedentes de la publicidad. Existe abundante bibliografía sobre la crítica de la economía política de la comunicación por el excesivo peso que tienen los anunciantes y el sistema político en el desarrollo mediático y, en especial, en la concepción de la televisión como un medio de masas. Ante esta situación, la naturaleza de la empresa se revela: son agentes de la responsabilidad social porque transmiten la información, ocupan el ocio y vehiculan las ideas, mientras que desarrollan una actividad económica industrial de acuerdo con los criterios clásicos de gestión.

No existe un acuerdo unánime sobre un determinado catálogo de medidas específicas para las empresas mediáticas, si bien se han planteado algunas necesidades tales como el equilibrio entre los derechos de voto en los consejos de administración y la dirección periodística y audiovisual de la empresa (Picard, 2005: 6). Estos mecanismos de control interno y externo separan explícitamente la vinculación directa entre propiedad y gerencia, de modo que ambos actores puedan desarrollar sus tareas de acuerdo con la misión encomendada. Más claro parecen los cuatro tipos básicos de conflicto de interés a los que se enfrentan las empresas periodísticas. A saber, el interés público, los consejeros cruzados, las propias actividades y productos del grupo empresarial y las actividades de la competencia (Arrese, 2006). Sucede que los conflictos acaban repercutiendo sobre las tareas informativas del periodista, que es el último eslabón de la cadena. La paradoja de la objetividad consiste en que el profesional cuenta con una credibilidad de partida, que le hace acreedor ante la sociedad de una responsabilidad informativa contrastada, templada e imparcial. Pero acontece que el 
periodista desempeña su trabajo en el marco de una organización que tiene que apoyar y dotar de instrumentos adecuados para el libre ejercicio de la profesión. La finalidad comercial de las empresas periodísticas estructura la identidad del periodista y puede minar la credibilidad informativa. Estos procesos y rituales de objetividad se materializan en prácticas, técnicas y códigos de conducta que generan un enfoque, un contexto y una socialización de la información. Pocos periodistas cuentan con una identidad consolidada e independiente que esté por encima de la identidad de la cadena.

La autocensura puede producirse por un exceso de confianza en la organización y por la consolidación de la espiral del silencio. Si un periodista considera que la emisión de una información audiovisual repercute negativamente en los propios intereses comerciales pero redunda en el interés público, ¿qué debe hacer? Este conflicto, marcado por la inestabilidad laboral y la más que posible conspiración de silencio, suele conducir a la no emisión en detrimento del interés público, el pluralismo y la opinión pública informada. Pasamos, entonces, de la función watchdog (guardián del interés público) a guard-dog (protector de los intereses comerciales de la organización).

El profesor Sánchez Tabernero (2008) ha analizado los principios y la medición de la calidad audiovisual. Sugiere que no es correcto identificar contenidos de calidad únicamente con aquellos de alta cultura, educación, sino que debe incluir la demanda y la preferencia explícita del consumidor. En el plano de la información audiovisual, la calidad exige el rigor, la profundidad, la comprensibilidad del mensaje, el interés humano, la corrección en el lenguaje, la pluralidad de fuentes, el respeto a la intimidad y la calidad técnica de las imágenes y el sonido, entre otros factores. Así, la mayoría de las empresas de televisión generalistas han apostado únicamente por un modelo económico basado en el audímetro, han confundido el producto con la necesidad que satisface. El proyecto empresarial ha desprestigiado algunas marcas y ha fomentado el contenido banal.

\section{LAS EMPRESAS ESPAÑOLAS DE TELEVISIÓN ANTE LA RSC}

Las experiencias en materia de responsabilidad social corporativa son diversas. Cada empresa define el concepto según su naturaleza e interés, por lo que no es sencillo elaborar un estudio comparativo que sea relevante desde el punto de vista científico. La redacción de las memorias condiciona el contenido de las mismas, ya que están elaboradas de acuerdo con los propios intereses. Es interesante valorar qué se aporta y define como RSC, pero resulta sumamente complicado contrastar algunas informaciones y datos. Por esa razón, las memorias se han empleado como referentes, pero no como documentos científicos.

\subsection{Antena 3 TV}

El informe anual de 2005 y 2006 (últimos disponibles en la red) recogen, bajo el epígrafe específico de "responsabilidad social", las acciones emprendidas en 
materia de RSC. Se define como una "política de cercanía" y "acciones solidarias" que dan "respuesta a las principales preocupaciones sociales de cada momento". Las acciones de 2005 son "Un puente solidario" (que recaudó 11 millones de euros para las acciones de Cruz Roja en Asia), "En la carretera, un poquito de por favor", "Díselo a mamá" (por el día de la madre), "Apaga y vámonos a estudiar" (selectividad), "Cierra el grifo" (sequía) y "Es fácil dejar de fumar". En la memoria de 2005 se destaca la firma del código de autorregulación sobre contenidos televisivos e infancia, junto con Gestevisión Telecinco, Sogecable y RTVE. Se explica el contenido del código, pero no se evalúa su grado de cumplimiento. En materia de formación continua, se explica el "Catálogo de formación", con 337 cursos destinados a la mejora de los conocimientos técnicos, los idiomas y otras habilidades. Se informa de que 1.866 empleados han recibido formación, con un promedio de 21 horas. El plan se completa con el "Plan de becarios", los acuerdos con dos universidades y sendos másteres específicos. El objetivo expreso es "acompañar la evaluación del negocio para optimizar la gestión del presente y afrontar con garantías el futuro". Finalmente, se informa de las actividades de la Fundación Antena 3, creada legalmente el 25 de noviembre de 2005. Se define como entidad privada sin ánimo de lucro que realiza actividades destinadas a la infancia y primera juventud, en especial para aquellos hospitalizados. La memoria de 2006 centra las actividades de RSC en la fundación (atención a padres de niños hospitalizados, creación del canal Fan3, entre otras). Completa el catálogo con la adhesión a la Carta Europea de Seguridad Vial, la iniciativa "Un libro, un juguete", el apoyo a la VII Campaña de Fracaso Escolar y la Gala Inocente, entre otras. En el ámbito de la formación, el catálogo recoge una oferta de 339 cursos, de los que se han impartido 158 talleres. 1.737 empleados han pasado por algún curso con una media de 20,6 horas. Se completa de nuevo con el plan de becarios y con la colaboración con dos universidades para cursos de posgrado. La novedad de la memoria 2006 es la introducción de un epígrafe dedicado al medio ambiente. En él, se explica en qué consiste el ahorro energético (gestión racional del consumo y renovación de la tecnología), el reciclaje de los residuos (papel y video). No se aportan cifras.

\subsection{Telecinco}

Esta empresa presenta el informe más completo y elaborado de entre las empresas privadas en España. Define la responsabilidad social corporativa como "un compromiso integral, que afecta a todas las dimensiones de la actividad de la cadena y a todas las relaciones que mantiene con los grupos de interés con los que se relaciona". Fruto de este planteamiento, la RSC se integra en la cadena de mando con una dirección que depende de la Secretaría General del Consejo. Se apuesta así por la sostenibilidad del proyecto y por la gestión a largo plazo. Con esta meta, Telecinco se ha adherido al Pacto Mundial de las Naciones Unidas y a la Global Reporting Initiative. Telecinco indica los grupos de interés, que señala como "pilares sobre los que se apoya la responsabilidad corporativa en la compañía" y ante quienes responde, y los canales de comunicación abiertos. Los grupos de interés se definen como "aquellas personas, colectivos o instituciones 
a los que afecta de forma significativa su actividad y que, a su vez, influyen en gran medida en los resultados obtenidos por la compañía". La última medida emprendida es la realización de una consulta a estos grupos para la elaboración del Informe 2007, todavía no disponible.

Los ocho grupos de interés y sus herramientas son las siguientes:

1) Empleados: canales de comunicación, condiciones laborales y formación;

2) Accionistas: transparencia, confianza, departamento de relación con los inversores;

3) Anunciantes: fuente principal de ingresos, Acuerdo de procedimiento para la comercialización y gestión de espacios publicitarios en Televisiones Nacionales Generalistas, Garantía de adjudicación de espacios (GARAD), racionalización del coste de acuerdo con el valor real, el valor añadido, la demanda y el nivel de servicio;

4) Audiencia: contenidos informativos, entretenimiento o publicitarios, criterios de honestidad, veracidad, independencia y responsabilidad, Códigos de Autocontrol y Código de Autorregulación;

5) Competidores: Acuerdo de buenas prácticas en materia publicitaria;

6) Proveedores: participaciones minoritarias en productoras;

7) Organismos de la administración pública: UTECA;

8) Organizaciones sociales: lazos con la sociedad civil, 12 meses, 12 causas, Fundación Empresa y Sociedad, AISGE o Asociación Española contra el cáncer.

La RSC de Telecinco se desarrolla en dos ámbitos. El primero se limita a su condición de empresa y es muy común en todos los sectores: el diálogo con los grupos de interés, la transparencia, el desarrollo social, económico y ambiental sostenible, la gestión responsable de los recursos humanos y el respeto a los derechos humanos e igualdad de oportunidades. El segundo ámbito se concreta en su condición de empresa audiovisual. Esta especificidad se perfila en dos objetivos: la identificación de las buenas prácticas relacionadas con el contexto de sostenibilidad entre las compañías del sector y la determinación de los asuntos relevantes concernientes a los medios audiovisuales. Se analizaron las memorias de sostenibilidad de las compañías europeas más avanzadas en RSC para determinar un catálogo de buenas prácticas y, en particular, de las dedicadas al sector audiovisual. Entre los referentes se cuentan el Media CSR Forum, el foro SustainAbility, la Unión de Televisiones Comerciales y Autocontrol.

Según el examen de Telecinco, los puntos más relevantes de los medios audiovisuales son los siguientes: independencia e imparcialidad; publicidad responsable; pluralidad y libertad de expresión; fomento de la cultura; capacidad de difusión de los retos relacionados con la sostenibilidad; protección de la infancia y la adolescencia; difusión de contenidos socialmente responsables. 
La plantilla se ha consolidado en 1.184 personas, el $98,2 \%$ con contratos indefinidos. La edad media es de 38 años y con experiencia en la compañía de 9,7 años. La distribución por sexos es equilibrada, con el $46 \%$ de mujeres, sobre todo en la redacción y en las jefaturas de departamento. La contratación temporal de personal para la producción se realiza a través de empresas de trabajo temporal. La política de contratación se define mediante la promoción y la incorporación de jóvenes profesionales con potencial de desarrollo. Se afirma este hecho por la conversión de 49 contratos temporales en fijos. En el ámbito de las relaciones laborales, el comité de empresa representa al $82,5 \%$ de la plantilla y el convenio colectivo ampara al $92,2 \%$ de la plantilla de Telecinco y Atlas España y al $75 \%$ del total de las empresas de la compañía. Se cuenta con un plan de prevención de riesgos laborales. En el plano de la formación continua, la cadena ha desarrollado 377 acciones, alcanzando el total de 36.659 horas para 736 participantes, así como un programa de prácticas para 275 universitarios. La comunicación interna se orienta hacia la integración y la motivación. Se publica la revista Punto de encuentro, el portal del empleado y los tablones de anuncios. También destaca la intranet y los desayunos de trabajo, que consisten en reuniones periódicas entre la dirección de la empresa y los departamentos.

La acción "12 meses, 12 causas" es gestionada por la Dirección de Comunicación y Relaciones Externas, dado su carácter de publicidad social y no de RSC en sentido estricto. Es un proyecto solidario cuyo objetivo es la concienciación acerca de temas que preocupan a la sociedad mediante la cesión de espacios para la emisión de spots. "Deja tu huella" ha sido una acción especial en 2006, junto con los proyectos de colaboración con la Dirección General de Tráfico, el Instituto de la Mujer o la Asociación Española contra el cáncer. El apoyo a la cultura se manifiesta a través de la producción cinematográfica, que gestiona la filial Telecinco Cinema (antiguos Estudios Picasso), y la colaboración con la AISGE, gestora de los derechos de propiedad intelectual de actores, dobladores, bailarines y directores de escena. La memoria de RSC obvia las obligaciones derivadas de la Ley del Cine.

En materia de medio ambiente, la memoria recoge las acciones acometidas tanto en la gestión interna de actividades diarias como en la capacidad de influir y concienciar a la audiencia. Las líneas de trabajo se relacionan con los compromisos ambientales a escala mundial como el Pacto Mundial de las Naciones Unidas. La idea general es la extensión de la ecoeficiencia, definida en el documento como "el proceso continuo de maximizar la productividad de los recursos, minimizando desechos y emisiones y generando valor para la empresa, sus clientes, sus accionistas y demás grupos de interés". Seguidamente se detallan los procesos y los resultados de los mismos. En materia de protección de datos, la cadena se ha adaptado a los requerimientos legales que se han sumado a otras medidas no obligatorias.

\subsection{Sogecable}

La empresa define la RSC como la incorporación voluntaria de los asuntos 
de orden social y medioambiental en la estrategia de negocio y en las relaciones con los agentes. La idea se compone de excelencia ética y moral, el respeto a un código de valores, la transparencia informativa, la acción social, el respeto al medio ambiente y la mejora de las relaciones laborales.

En relación con la transparencia informativa, Sogecable destaca el grado de cumplimiento del Convenio de Autorregulación de la Publicidad en Televisión y el código de conducta periodística, que afecta a los servicios informativos de Cuatro y al conjunto de actividades de producción y distribución de CNN+. Lo interesante no es tanto el contenido del código (principios periodísticos al uso, vistos y leídos en numerosos documentos), sino su inclusión y publicación tanto en la memoria como la página web al alcance de la comunidad de espectadores.

Los trabajadores han desarrollado desde 1993 el Proyecto Cero 25, que consiste en la donación voluntaria del $0,25 \%$ de la nómina para la financiación de proyectores de cooperación al desarrollo y protección del medio ambiente. Las ONG beneficiadas han sido Fundación Prodis (financiación becas niños discapacitados psíquicos), Farmamundi (compra de medicinas refugiados saharauis en Tinduf), Médicos sin Fronteras (Acción integral Proyecto Busía), Ingenieros sin Fronteras (proyecto hidrosanitario en Tanzania), A tiempo (reforma de aula de informática), Mundo Cooperante (hogar materno infantil en Cartagena de Indias), ACNUR (repatriación y reintegración refugiadas congoleñas), Aldeas Infantiles de Cuenca y Casas de la Esperanza en Granada. La acción se completa con el "Día Solidario", que dona la nómina de un día para un proyecto concreto. Otras acciones solidarias específicas son la emisión de contenidos dedicados a la promoción de los derechos humanos ("Humania" en Documanía) y temas sociales ("Callejeros" en Cuatro). Por último, se incide en la publicidad social y la cesión gratuita de espacios para ONG e instituciones de reconocido prestigio.

En relación con la protección del medio ambiente, Sogecable ha preparado la "Guía de buenas prácticas medioambientales" y ha emprendido un conjunto de acciones para la reducción, el reciclaje y la reutilización de energías. La memoria detalla los resultados. El apoyo a la cultura se vehicula a través de Sogecine, que apoya el desarrollo del cine español (90 películas en 16 años, inversión anual del $5 \%$ de los beneficios y un canal dedicado a la producción nacional), y el deporte (derechos de emisión, campeonato infantil de fútbol 7). Asimismo, se pone en valor las relaciones y la política de apoyo y patrocinio. Entre los beneficiados, se encuentran el Festival de Cine de Málaga, el Liceo de Barcelona, la Academia de Televisión, la Fundación Escuela de Periodismo, la Asociación de Amistad hispano-francesa, y la Asociación de la Prensa de Madrid.

\section{HACIA LA DEFINICIÓN DE UN NUEVO MODELO DE GESTIÓN}

La empresa periodística tiene que desarrollar las estrategias de responsabilidad social corporativa adecuadas a la misión, la visión y el modelo de negocio específico. La mayoría de las acciones desarrolladas por otros sectores (medio 
ambiente, derechos laborales) cuadran perfectamente con la doble condición de actividad económica y cultural. Falta completar los indicadores exclusivos, como sucede en otros países del entorno.

Las experiencias españolas son pobres, ya que no han comprendido el alcance y la función de la responsabilidad social corporativa. En los tres casos analizados no se parte de una definición propia acorde con la bibliografía disponible. Al contrario, se caracterizan por la imprecisión y la vaguedad semántica. Según se deduce de la lectura de las memorias y los informes de gestión, es una herramienta menor, cajón de sastre para todas las acciones que no tienen cabida en otras áreas de la gestión. La Sexta y TVE son dos casos relevantes. A fecha de hoy, ninguna de las dos compañías ha puesto a disposición pública sus informes y sus memorias. Si existen, no son fácilmente accesibles, luego tampoco cumplen con el criterio básico de transparencia informativa que sustenta el nuevo estilo de gestión.

Las tres televisiones privadas expuestas reducen el capítulo básicamente a la publicidad social, las acciones de filantropía y las horas de formación interna para los empleados. Esto no es la responsabilidad social corporativa que consiste en la redefinición de la gestión de la empresa. Está directamente ligada a las operaciones, las inversiones y las decisiones empresariales. El marketing y la publicidad social son herramientas, pero no fines de la RSC, que se fundamenta en los departamentos de control de la gestión, riesgo, transparencia, comunicación y rendición de cuentas.

Urge que se desarrollen las estrategias propias del sector. Las herramientas para conseguir el objetivo son conocidas: manuales y libros de estilo, transparencia ante el conflicto de intereses, la defensa de la libertad de expresión, el autocontrol profesional, los códigos deontológicos, la normalización del trabajo periodístico y el respeto al idioma. En el plano individual, el periodista tiene que contar efectivamente con las herramientas legales que son la cláusula de conciencia y el secreto profesional. Ambas tienen como finalidad asegurar la dimensión pública/cívica de la información: no es sólo un derecho propio, sino ejecutado en beneficio de la sociedad.

\section{REFERENCIAS BIBLIOGRÁFICAS}

ARRESE, Á.: "Reflexiones sobre el buen gobierno corporativo en las empresas periodísticas". Doxa Comunicación (2006), n 4, pp. 59-82.

CARROLL, A.; y BUCHHOLZ, A. K. (1999): Business \& Society. Ethics \& Stakeholders Management. $5^{\mathrm{a}}$ edición. Cincinnati: South Western College Publishing.

CUBELES, X.: "Calidad y televisión: consideraciones desde la óptica del mercado". Quaderns del CAC (2002), no 13, pp.27-39.

PICARD, R. G. (2005): Corporate Governance of Media Companies. Jönköping: Jönköping International Business School. 
SÁNCHEZ TABERNERO, A. (2008): Los contenidos de los medios de comunicación: calidad, rentabilidad y competencia. Barcelona: Deusto.

SOLOSKI, J. et al. (2001): Taking stock: Journalism and the Publicly Traded Newspaper Company. Ames: lowa State University Press.

SOLOSKI, J.: Taking Stock Redux: Corporate Ownership and Journalism of Publicly Traded Newspaper Companies. En PICARD, R. G. (2005): Corporate Governance of Media Companies. Jönköping: Jönköping International Business School, pp.59-76.

\section{Breve semblanza biográfica del autor}

Juan Luis Manfredi Sánchez es profesor de IE Universidad y doctor en Comunicación por la Universidad de Sevilla, donde obtuvo las licenciaturas en Periodismo e Historia. MBA candidate en IE Business School y máster en Gestión de Empresas Audiovisuales y en Administraciones Públicas por la misma Universidad. Ha obtenido los premios Blas Infante, RTVA y SGAE Fundación Autor por trabajos escritos acerca de la televisión, los mercados audiovisuales y la comunicación internacional.

(Recibido el 12-03-2009, aceptado el 03-06-2009) 